\title{
Posteromedial Dislocation of the Elbow with Lateral Condyle Fracture in a Six-Year-Old Boy
}

\section{Ahmad Khaled Almigdad*, Ghandi Almanasir, Fahed Alsarhan, Mohammad Alsaideh and Khaled Bani Melhem}

Department of Orthopedic Surgery, Royal Medical Services, Jordan

*Corresponding Author: Ahmad Khaled Almigdad, Department of Orthopedic, Royal Medical Services, Amman, Jordan.
Received: June 05, 2021

Published: June 17, 2021

(C) All rights are reserved by Ahmad Khaled

Almigdad., et al.

\begin{abstract}
Traumatic elbow dislocation is a rare injury in children and usually occurs at older ages. Elbow dislocation is classified based on the proximal radio-ulnar joint position in relation to the distal humerus. Most elbow dislocations in children are posterolateral and might be associated with medial humeral condyle fracture. Posteromedial dislocation is extremely rare.

This article describes a six-year-old boy with posteromedial dislocation of the elbow associated with a displaced fracture of the lateral humeral condyle. Dislocation is closely reduced at the emergency room, and the lateral condyle fracture is subsequently fixed at the operating room by Kirschner wires through a lateral approach. A long arm cast was applied for four weeks then physiotherapy was initiated. The child was followed at 3 and 6 months to evaluate the outcome.
\end{abstract}

Keywords: Elbow Dislocation; Pediatric; Lateral Condyle Fracture

\section{Introduction}

Traumatic elbow dislocation accounts for $3-6 \%$ of pediatric elbow injuries $[1,2]$. It occurs in the second decade of life, while the dislocation in younger age groups is extremely rare $[3,4]$. Elbow dislocation is classified based on the position of the proximal radio-ulnar joint in relation to the distal humerus; the majority (95\%) of elbow dislocations are posterior, and $70 \%$ out of them are posterolateral dislocation $[5,6]$. In addition, elbow dislocation might be associated with other injuries as medial epicondyle fracture, brachial artery, and median nerve injury [7-9].

In this study, we present a six-year-old boy with posteromedial elbow dislocation and displaced lateral condyle fracture.

\section{Case Report}

A six-year-old boy had presented to the emergency department one hour after falling on his outstretched right hand. Clinical evaluation of the child revealed gross deformity of the right elbow and intact neurovascular examination of the limb. There were no other injuries. A radiograph of the limb showed dissociation of trochlearulnar articulation and intact radio-capitellar joint (Figure 1). The whole distal fragment was displaced poster-medially. The dislocation was closely reduced in the emergency room, and long arm cast was applied, and a neurovascular exam was rechecked after reduction. Post reduction radiograph showed reduced elbow with lateral condyle fracture. An Elbow CT scan was performed post-reduction to better understand the fracture pattern and confirm the conventional radiograph's initial impression.

The child was operated on the next morning and the lateral condyle fracture was fixed through lateral elbow approach. Fracture fragments were fixed by two Kirschner wires and the stability of elbow was checked (Figure 2). Elbow was stable through full range of movement; therefore, long arm cast was applied. Wound checked at two weeks and cast reapplied for other two weeks.

At four weeks, elbow radiographs demonstrated healed fracture. Therefore, both cast and Kirschner wires were removed, and elbow range of movement was initiated. 


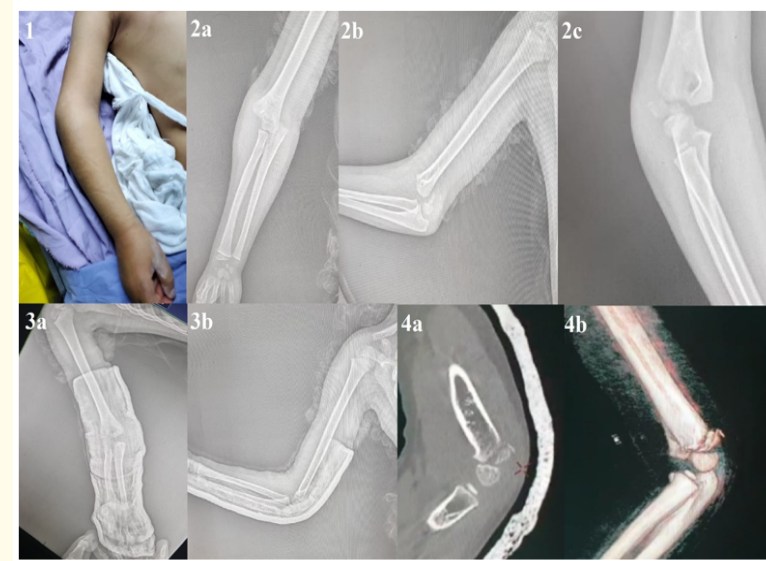

Figure 1: a: Clinical and radiological picture of a 6-year old boy with right posteromedial elbow dislocation associated with lateral humeral condylar fracture. (1) elbow clinical presentation. (2a, 2b and 2c) pre-reduction elbow radiograph.

( $3 \mathrm{a}$ and $3 \mathrm{~b}$ ) post-reduction elbow radiograph. (4a and $4 \mathrm{~b}$ ) post-reduction elbow CT scan show reduced elbow with lateral condyle fracture.

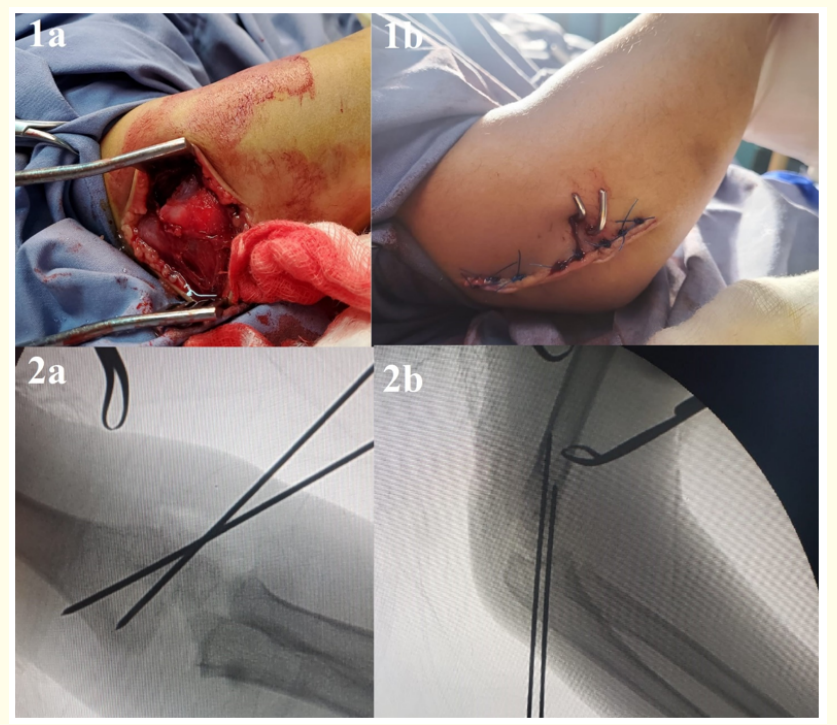

Figure 2: Operative treatment of lateral condyle fracture associated with a posteromedial dislocation. (1a and $1 \mathrm{~b}$ ) lateral elbow approach for fracture reduction and Kirschner wire fixation of the fracture. ( $2 a$ and $2 b$ ) Kirschner wires configuration for lateral condyle fracture fixation.
The child was followed regularly by a physiotherapist and evaluated monthly at the clinic for the first three months. At six months follow up, the child regains arc of flexion-extension from full extension $\left(0^{\circ}\right)$ to $140^{\circ}$ flexion; (left elbow was $-20^{\circ}$ in extension, due to ligamentous laxity to $145^{\circ}$ flexion). Pronation- supination arc was symmetrical at both sides $\left(85^{\circ}\right.$ supination and $75^{\circ}$ pronation) (Figure 3). Elbow radiograph showed anatomically healed fracture with no signs of avascular necrosis or any other abnormality (Figure 4).

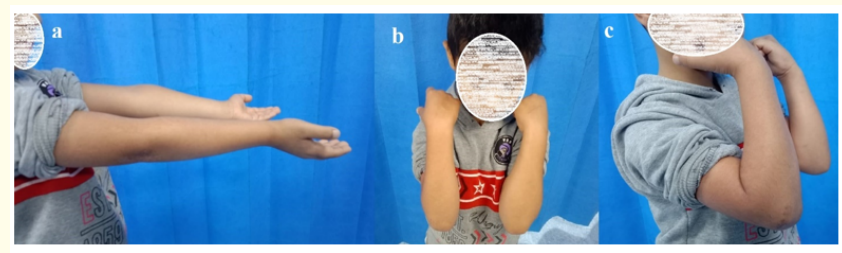

Figure 3: Six months- postoperative clinical picture. (a) right elbow extension $-20^{\circ}$ due to hyperlaxity compared to $0^{\circ}$ in the injured elbow. (b, c) right elbow flexion $145^{\circ}$ compared to $140^{\circ}$ in the left elbow.

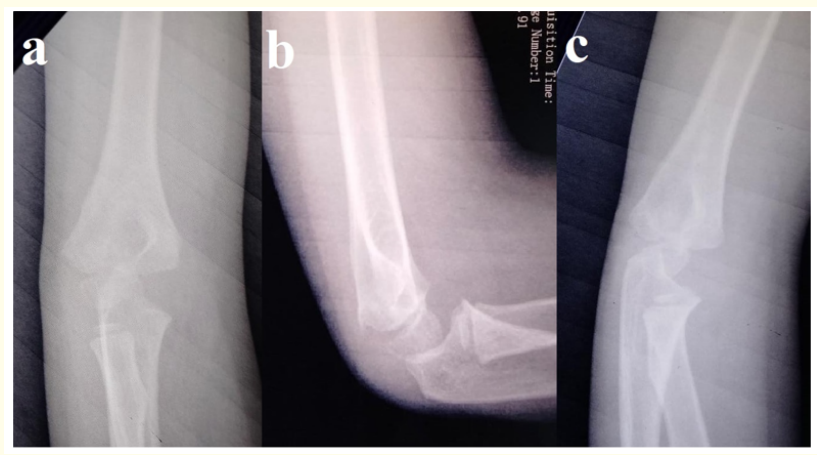

Figure 4: Six months- follow-up radiographs showed healed fracture and reduced radio-capitellar and unlno-trochlear joints. (a) anterioposterior view. (b) lateral view. (c) oblique view.

\section{Discussion}

Although elbow dislocation is the most common dislocation in children, it still a rare injury. Lateral condyle fracture is a common pediatric injury, but an association with posteromedial dislocation is an extremely rare injury in children. Therefore, there are only 
a few cases reported in the literature. Kirkos JM., et al. reviewed four patients with posteromedial dislocation associated with lateral condyle fracture. The average duration of follow-up was seven years and six months (range, 3 - 13 years). All patients were treated by closed reduction of the elbow dislocation, and the lateral humeral condyle fracture was fixed through the lateral approach by Kirschner wires. Three patients had excellent results, and the fourth patient had good results [10].

Cheng PG., et al. reviewed three patients, one case treated closely for lateral condyle fracture and complicated by non-union. The second case developed malunion secondary to inadequate reduction regardless of open reduction; both cases had poor functional results. Nevertheless, the authors recommended close reduction for the dislocation and open reduction for direct visualization and anatomical reduction of lateral condyle fracture to avoid poor outcomes [11].

Lane., et al. compared the surgical findings and clinical outcomes of 21 patients with lateral condylar fractures. In eight patients, their fractures were associated with posteromedial elbow dislocation. There were no significant differences in bone union time and complication rate between isolated lateral condyle fracture groups and patients with combined lateral condyle fracture and posteromedial dislocation. Nevertheless, the rehabilitation time for dislocation-associated fracture was longer because the soft tissue injury was worse than isolated lateral condyle fracture [10].

\section{Conclusion}

Pediatric posteromedial elbow dislocation with lateral condyle fracture is extremely rare. According to our experience and literature review, this injury mandates immediate closed reduction of the dislocation and open reduction of the associated lateral condyle fracture to regain joint congruency and elbow stability. Therefore, better outcomes and lower complications can be achieved. Combined elbow dislocation and fractures are expected to need longer rehabilitation time. Therefore, compliance with physiotherapy is vital to regaining elbow function.

\section{Acknowledgment}

The authors thank the child H.M.Z and his father M.M.Z for their cooperation and participation in this study.

\section{Ethical Consideration}

Written consent for surgery and verbal one for the study were obtained. In addition, the patient's father has given his consent for the child's images and other clinical information to be reported in the journal. The father understands that his child's name and initials will not be published, and due efforts will be made to conceal the identity, but anonymity cannot be guaranteed.

\section{Ethical Approval}

This study has been approved by the local ethical committee of the Jordanian Royal Medical Services under study ID: RMS 4-2021:3 on May $27^{\text {th }} 2021$.

\section{Conflict of Interest}

There are no conflicts of interest.

\section{Financial Support and Sponsorship}

This study did not receive any specific grant from funding agencies in the public, commercial, or not-for-profit sectors.

\section{Bibliography}

1. Wilkins KE. "Dislocations of Joints of the Elbow". In Rockwood CA, Wilkins KE, King RE (eds). Fractures in Children. Philadelphia, JB Lippincott Company 3 (1991): 532-554.

2. Hyvönen H., et al. "Recent trends in children's elbow dislocation with or without a concomitant fracture". BMC Musculoskeletal Disorders 20.1 (2019): 294.

3. Alexander MG and Graham HK. "Dislocations of the elbow in children". In: Stanley D, Trail I, editors. Operative elbow surgery. Philadelphia: Elsevier 4 (2012): 203-222.

4. Kim HH and Gauguet JM. "Pediatric Elbow Injuries". Semin Ultrasound CT MR 39.4 (2018): 384-396.

5. Lieber J., et al. "Acute traumatic posterior elbow dislocation in children". Journal of Pediatric Orthopaedics B 21.5 (2012): 474-481.

6. Little KJ. "Elbow fractures and dislocations". Orthopedic Clinics of North America 45.3 (2014): 327-340.

7. Sharma H., et al. "Complex pediatric elbow injury: an uncommon case”. BMC Musculoskeletal Disorders 6 (2005): 13.

8. Combourieu B., et al. "Pediatric elbow dislocation associated with proximal radioulnar translocation: a report of three cases and a review of the literature". The Journal of Bone and Joint Surgery American 92.8 (2010): 1780-1785.

9. Silva M., et al. "Elbow dislocation with an associated lateral condyle fracture of the humerus: a rare occurrence in the 
pediatric population". Journal of Pediatric Orthopaedics 35.4

(2015): 329-333.

10. Kirkos JM., et al. "Posteromedial dislocation of the elbow with lateral condyle fracture in children". Clinical Orthopaedics and Related Research 408 (2003): 232-236.

11. Cheng PG., et al. "Posteromedial dislocation of the elbow with lateral condyle fracture in children". Journal of the Chinese Medical Association 72.2 (2009): 103-107.

12. Lan X., et al. "Comparative study of lateral condyle fracture with or without posteromedial elbow dislocation in children". International Orthopaedics 42.3 (2018): 619-624.

Volume 4 Issue 7 July 2021

(c) All rights are reserved by Ahmad Khaled Almigdad., et al. 